\title{
Exterior Dirichlet Problem for Translating Solutions of Gauss Curvature Flow in Minkowski Space
}

\author{
Hongjie Ju \\ School of Science, Beijing University of Posts and Telecommunications, Beijing 100876, China \\ Correspondence should be addressed to Hongjie Ju; hjju@bupt.edu.cn
}

Received 20 June 2014; Accepted 30 July 2014; Published 12 August 2014

Academic Editor: Sining Zheng

Copyright (C) 2014 Hongjie Ju. This is an open access article distributed under the Creative Commons Attribution License, which permits unrestricted use, distribution, and reproduction in any medium, provided the original work is properly cited.

We prove the existence of solutions to a class of Monge-Ampère equations on exterior domains in $\mathbb{R}^{n}(n \geq 2)$ and the solutions are close to a cone. This problem comes from the study of the flow by powers of Gauss curvature in Minkowski space.

\section{Introduction and Main Results}

The Euclidean space $\mathbb{R}^{n+1}$ endowed with the Lorentz metric $d s^{2}=\sum_{i=1}^{n} d x_{i}^{2}-d x_{n+1}^{2}$ is called Minkowski space. We denote it by $\mathbb{R}^{n, 1}$. A space-like hypersurface in $\mathbb{R}^{n, 1}$ is a Riemanian $n$-manifold, having an everywhere lightlike normal field $v$ which we assume to be future directed and thus satisfy the condition $\langle\nu, v\rangle=-1$. Locally, such surfaces can be expressed as graphs of functions $x_{n+1}=u\left(x_{1}, \ldots, x_{n}\right): \mathbb{R}^{n} \rightarrow \mathbb{R}$ satisfying the space-like condition $|D u(x)|<1$ for all $x \in \mathbb{R}^{n}$.

If a family of space-like hypersurfaces $X_{t}=X(\cdot, t)$ : $\mathbb{R}^{n} \rightarrow \mathbb{R}^{n, 1}$ satisfies the evolution equation

$$
\frac{\partial X}{\partial t}=-K^{\alpha} v
$$

on some time interval, we say that the surfaces $M_{t}:=X_{t}(M)$ are evolved by $K^{\alpha}$-flow, where $K(\cdot, t)$ is the Gauss curvature of $M_{t}$ and $\alpha \neq 0$ is a constant. When the initial surface is a graph over a domain $\Omega \subset \mathbb{R}^{n}$, (1) is equivalent, up to a diffeomorphism in $\mathbb{R}^{n}$, to

$$
\frac{\partial V}{\partial t}=\sqrt{1-|D V|^{2}}\left[\frac{\operatorname{det}\left(D^{2} V\right)}{\left(1-|D V|^{2}\right)^{(n+2) / 2}}\right]^{\alpha}
$$

with $|D V(\cdot, t)|<1$, where $V$ is a function defined in $\Omega \times[0, T)$.

The flow (2) was studied in [1] for the special case $\alpha=$ 1. In fact, the authors in [1] used the flow (2) to prove existence and stability of smooth entire strictly convex spacelike hypersurfaces of prescribed Gauss curvature and give a new proof of Theorem 3.5 in [2].

A function $u=u(x)$ is called a translating solution to the $K^{\alpha}$-flow if the function $V(x, t)=u(x)+t$ solves (2). Equivalently, $u(x)$ is an initial hypersurface satisfying

$$
\operatorname{det}\left(D^{2} u\right)=\left(1-|D u|^{2}\right)^{((n+2-(1 / \alpha)) / 2)} .
$$

The space-like condition reads as

$$
|D u(x)|<1 .
$$

The space-like hypersurfaces evolved by mean curvature flow in Minkowski space were studied in [3-6]. The translating solutions were introduced in $[3,4]$ and studied in $[7,8]$.

In this paper, we consider strictly convex space-like hypersurfaces of translating solutions to $K^{\alpha}$-flow as graphs over $\mathbb{R}^{n} \backslash D$, where $D \subset \mathbb{R}^{n}$ is an open domain whose boundary $\partial D$ is a smooth submanifold of $\mathbb{R}^{n}$. We want to look for a function $u \in C^{\infty}\left(\mathbb{R}^{n} \backslash D\right)$, which solves the problem (3)(4) with the boundary condition

$$
u=\phi \quad \text { on } \partial D,
$$

where $\phi \in C^{\infty}(\partial D)$ is a given function.

There are similar problems for the equation of translating solution of Gauss curvature flow in Eucliden space [9], the equation of prescribed Gauss curvature in Eucliden space [10], and the equation of prescribed Gauss curvature in 
Minkowski space [11], respectively. It was shown that there are convex solutions to the Dirichlet problems for the three equations on exterior domains, and the solution is close to the rotationally symmetric one at infinity for the first equation and close to a cone for the second and third equation under the assumption that there exists a strictly convex subsolution which is close to a cone up to the third order (see (7) and (8)).

In this paper, we will show that the same results as in $[10,11]$ hold for the problem (3)-(5). We would like to point out that (3) is essentially different from the equations in [911]. For example, the equation of prescribed Gauss curvature in Minkowski space, $\operatorname{det}\left(D^{2} u\right)=\left(1-|D u|^{2}\right)^{(n+2) / 2}$, has an explicit solution $u=\sqrt{1+|x|^{2}}$, from which one can easily construct subsolution or supersolution for given Dirichlet problems. However, it is unknown if there is such a solution to (3). In particular, it has no solution in the form of $u=$ $\left(1+|x|^{2}\right)^{\gamma}$.

Definition 1. A function $\underline{u} \in C^{\infty}\left(\mathbb{R}^{n} \backslash D\right)$ is called a subsolution of (3)-(5), if $\underline{u}$ is strictly convex and satisfies

$$
\begin{gathered}
\operatorname{det}\left(D^{2} \underline{u}\right) \geq\left(1-|D \underline{u}|^{2}\right)^{(n+2-\beta) / 2}, \quad \text { in } \mathbb{R}^{n} \backslash \bar{D}, \\
|D \underline{u}|<1, \quad \text { in } \mathbb{R}^{n} \backslash \bar{D}, \\
\underline{u}=\phi, \quad \text { on } \partial D .
\end{gathered}
$$

Here and below, we set $\beta=1 / \alpha$.

The main result of this paper is the following theorem.

Theorem 2. Let $D \subset \mathbb{R}^{n}(n \geq 2)$ be an open set whose boundary $\partial D$ is a smooth submanifold of $\mathbb{R}^{n}$ and $\phi \in C^{\infty}(\partial D)$. Suppose that $\beta<(3 / 2)-2 n$ and $\underline{u} \in C^{\infty}\left(\mathbb{R}^{n} \backslash D\right)$ is a subsolution of (3)-(5) which is close to a cone, that is,

$$
\sup _{\mathbb{R}^{n} \backslash D}|\underline{u}-| x||<\infty
$$

and satisfies the following decay conditions at infinity:

$$
\begin{gathered}
|D(\underline{u}-|x|)|=O\left(\frac{1}{|x|}\right), \\
\left|D^{2}(\underline{u}-|x|)\right|+\left|D^{3} \underline{u}\right|=O\left(\frac{1}{|x|^{2}}\right) .
\end{gathered}
$$

Then there exists a smooth, strictly convex hypersurface of the exterior Dirichlet problem (3)-(5) and the solution $u$ is close to a cone in the sense that

$$
\sup _{\mathbb{R}^{n} \backslash D}|u-| x||<\infty .
$$

Although the above theorem has an obvious disadvantage that it assumes the existence of a locally strictly convex subsolution, this assumption is reasonable and necessary in some case for the Dirichlet problems on nonconvex domains; see [12] for the details. However, in the special case when $D=B_{\rho_{0}}(0)$ is a ball and the boundary values are zero, we can construct an explicit subsolution.
Theorem 3. Let $D=B_{\rho_{0}}(0)$ with $\rho_{0}>0$ and $\phi \equiv 0$. If $\beta \leq 0$, then there is a strictly convex subsolution $\underline{u}$ of (3)-(5) such that (7) and (8) are satisfied.

We consider the local problem

$$
\begin{gathered}
\operatorname{det}\left(D^{2} u^{R}\right)=\left(1-\left|D u^{R}\right|^{2}\right)^{(n+2-\beta) / 2}, \quad \text { in } B_{R} \backslash \bar{D}, \\
\sup _{B_{R} \backslash \bar{D}}\left|D u^{R}\right|<1, \\
u^{R}=\underline{u}, \quad \text { on } \partial D \cup \partial B_{R},
\end{gathered}
$$

where $R>4 R_{0}$ and $D \subset B_{R_{0}}$ for some constant $R_{0}>$ 1. It is well known from the standard continuity method as in [13] that the Dirichlet problem (10) has a locally strict convex solution in $C^{\infty}\left(\bar{B}_{R} \backslash D\right)$. Our main task is to show that the $C^{2}$-norms of $u^{R}$ are uniformly bounded in $R$. Once this is established, by the standard Krylov/Shafanov theory, Schauder regularity theory, and a diagonal sequence argument, we can obtain a smooth locally strictly convex solution $u$ to (3)-(5) on exterior domain $\mathbb{R}^{n} \backslash D$.

The paper is organized as follows. In Section 2, we prove the $C^{0}$ and $C^{1}$ a priori estimates for $u^{R}$. The $C^{2}$-estimates are given in Section 3. Finally, we prove Theorem 3 in the last section.

\section{2. $C^{0}$ and $C^{1} \mathbf{A}$ Priori Estimates}

From now on, we assume $D$ and $\underline{u}$ as in Theorem 2 and $u^{R}$ as in (10); lower indices denote partial derivatives in $\mathbb{R}^{n}$, for example, $u_{i}=\partial u / \partial x_{i}$. The inverse of the Hessian of $u$ is denoted by $\left(u^{i j}\right)=\left(u_{i j}\right)^{-1}$. We use the Einstein summation convention. The letter $c$ denotes a constant independent of $R$ which may change its value from line to line throughout the text.

Without loss of generality we can assume that $0 \in D$. It is easy to check that $\bar{u}=\sqrt{1+|x|^{2}}+L$ is a supersolution to (3) for $\alpha<0$, where the constant $L>\max _{\partial D}\left(\phi-\sqrt{1+|x|^{2}}\right)$.

Owing to the maximum principle, we can obtain the following lemma as Lemma 2.2 in [10].

Lemma 4. The functions $u^{R}$ converge locally uniformly to a continuous function $u$ as $R \rightarrow \infty$. Moreover, $\underline{u} \leq u \leq \bar{u}$ in $B_{R} \backslash D$.

Proof. From the maximum principle we obtain that

$$
\underline{u} \leq u^{R} \leq \bar{u} \quad \text { in } B_{R} \backslash D
$$

for any $R>4 R_{0}$ and

$$
u^{R_{1}}=\underline{u} \leq u^{R_{2}} \quad \text { on } \partial B_{R_{1}} \cup \partial D
$$

for $4 R_{0}<R_{1}<R_{2}$. Again by the maximum principle, we have

$$
u^{R_{1}} \leq u^{R_{2}} \quad \text { in } B_{R_{1}} \backslash D .
$$


We conclude that $u^{R}$ are monotone in $R$ and converge locally uniformly to a continuous function $u$ according to Dini's theorem.

To simplify the notation, we will omit the index $R$ and from now on assume that $u$ is a solution of (10) with $R$ fixed sufficiently large. The estimate for the first derivatives is stated in the following lemma.

Lemma 5. For $R / 2 \leq|x| \leq R$, there is a constant $c$ independent of $R$ such that

$$
\begin{gathered}
\left|\nabla_{v}(u-\underline{u})(x)\right| \leq \frac{c}{R}, \\
\left|\nabla_{\tau} u(x)\right| \leq \frac{c}{\sqrt{R}}, \\
\sqrt{1-\frac{c}{R}} \leq|D u(x)|<1,
\end{gathered}
$$

where $\nu=x /|x|$ and $\tau$ are unit vectors parallel and orthogonal to $x$, respectively.

Proof. From the convexity of $u$ and Lemma 4, we can prove (14) and (15) by using the similar proof techniques of (2.2) and (2.3) in [10]. Then, we need only to prove (16). Since $u$ is strictly convex, for $R / 2 \leq|x| \leq R,|D u|$ attains its maximum at $\partial B_{R}$. In view of (8), we may take

$$
|D \underline{u}|^{2}=O\left(1-\frac{c}{R}\right) \text {. }
$$

Hence for $x \in \partial B_{R}$, by (14) and (17) we have

$$
\begin{aligned}
|D u(x)|^{2} & =\left|\nabla_{\tau} u(x)\right|^{2}+\left|\nabla_{\nu} u(x)\right|^{2} \\
& =\left|\nabla_{\tau} \underline{u}(x)\right|^{2}+\left|\nabla_{\nu} \underline{u}(x)+\nabla_{\nu}(u-\underline{u})(x)\right|^{2} \\
& \geq|D \underline{u}(x)|^{2}-2\left|\nabla_{\nu}(u-\underline{u})(x)\right| \geq 1-\frac{c}{R} .
\end{aligned}
$$

On the other hand, by the proof of Theorem 4.1 in [12],

$$
\max _{\partial B_{R}}|D u| \leq \max _{\partial B_{R}}|D \underline{u}|<1 .
$$

The lemma is completed.

\section{3. $C^{2}$ A Priori Estimates}

In this section, we prove the $C^{2}$ a priori estimates for solutions of (10) under the assumption of Theorem 2. As in [12], one obtains that the second derivatives on $\partial D$ are bounded uniformly in $R$. Furthermore, by considering the function

$$
w=\frac{a}{2}|D u|^{2}+\log u_{\xi \xi}
$$

for some constant $a>0$ and assuming its maximum over $(x, \xi) \in \overline{B_{R} \backslash D} \times S^{n-1}$ is attained at an interior, one can prove that

$$
\max _{B_{R} \backslash \bar{D}}|D u|^{2} \leq c+\max _{\partial B_{R} \cup \partial D}\left|D^{2} u\right|
$$

Therefore, it suffices to bound $\left|D^{2} u\right|$ on the outer boundary $\partial B_{R}$.

Next, we will give estimates for the tangential second derivatives, the mixed second derivatives, and the normal second derivatives on the outer boundary $\partial B_{R}$, respectively.

Theorem 6 (tangential second derivatives at the outer boundary). Let $x_{0} \in \partial B_{R}$ and $\tau_{1}, \tau_{2}$ be tangential directions at $x_{0}$. Then we have at $x_{0}$,

$$
\left.\left|u_{\tau_{1} \tau_{2}}-\right| x\right|_{\tau_{1} \tau_{2}} \mid \leq \frac{c}{R^{2}} .
$$

Proof. We may assume that $x_{0}=R \cdot e_{n} \equiv R \cdot(0, \ldots, 0,1)$. Then $\partial B_{R}$ is represented locally as graph of $\omega$, where

$$
\omega(\widehat{x})=\sqrt{R^{2}-|\widehat{x}|^{2}}, \quad \widehat{x}=\left(x_{1}, x_{2}, \ldots, x_{n-1}\right) \in \mathbb{R}^{n-1} .
$$

Note that the Dirichlet boundary condition implies

$$
(u-\underline{u})(\widehat{x}, \omega(\widehat{x}))=0 \text {. }
$$

We differentiate twice with respect to $\widehat{x}_{i}, \widehat{x}_{j}, 1 \leq i, j \leq n-1$ to obtain that, at $x_{0}$,

$$
(u-\underline{u})_{i j}+(u-\underline{u})_{n} \omega_{i j}+2(u-\underline{u})_{n j} \omega_{i}=0 .
$$

According to the decay conditions at infinity (8), we have $\left.\left|\underline{u}_{i j}-\right| x\right|_{i j} \mid=O\left(1 / R^{2}\right)$. Observing that

$$
w_{i}\left(x_{0}\right)=0, \quad \omega_{i j}\left(x_{0}\right)=-\frac{\delta_{i j}}{R} .
$$

Then, by Lemma 5 we have

$$
\left.\left|u_{i j}-\right| x\right|_{i j}|=|-(u-\underline{u})_{n} \omega_{i j}+(\underline{u}-|x|)_{i j} \mid \leq \frac{c}{R^{2}} .
$$

Theorem 7 (mixed second derivatives at the outer boundary). For $x_{0} \in \partial B_{R}$, let $\tau, v$ be unit vectors in tangential and normal directions, respectively. Then

$$
\left|u_{\tau \nu}\left(x_{0}\right)\right| \leq \frac{c}{\sqrt{R}}
$$

The proof is going to be put in three lemmas and will be finished below Lemma 10. Similar to Theorem 6, we may assume that $x_{0}=R \cdot e_{n}$ and represent $\partial B_{R}$ locally as graph of $\omega$ with $\omega(\hat{x})=\sqrt{R^{2}-|\widehat{x}|^{2}}$. We take the logarithm of (3),

$$
\log \operatorname{det} u_{i j}-\frac{n+2-\beta}{2} \log \left(1-|D u|^{2}\right)=0 \text {, }
$$

and differentiate with respect to $x_{k}$,

$$
u^{i j} u_{i j k}+\frac{n+2-\beta}{1-|D u|^{2}} u_{i} u_{i k}=0 \text {, }
$$


where $\left(u^{i j}\right)=\left(u_{i j}\right)^{-1}$. We introduce the linear differential operator $L$ by

$$
L w:=u^{i j} w_{i j}+\frac{n+2-\beta}{1-|D u|^{2}} u_{i} w_{i}
$$

and define the linear operator for $t<n$ :

$$
T:=\frac{\partial}{\partial x_{t}}+\sum_{\gamma=1}^{n-1} \omega_{\operatorname{tr}}(0) x_{\gamma} \frac{\partial}{\partial x_{n}} \equiv \frac{\partial}{\partial x_{t}}-\frac{x_{t}}{R} \frac{\partial}{\partial x_{n}} .
$$

In the following we restrict attention to the domain $\Omega_{\delta}:=$ $B_{\delta}\left(x_{0}\right) \cap B_{R}$ with $x_{0} \in \partial B_{R}$ and $\delta \leq R / 2$. Notice that $\Omega_{\delta} \subset$ $B_{R} \backslash \bar{D}$.

Lemma 8. The function $u-\underline{u}$ satisfies the following estimates:

$$
\begin{gathered}
|T(u-\underline{u})| \leq \frac{c}{\sqrt{R}} \quad \text { in } \Omega_{\delta}, \\
|T(u-\underline{u})| \leq \frac{c}{R^{2}}\left|x-x_{0}\right|^{2} \quad \text { on } \partial B_{R}, \\
|L T(u-\underline{u})| \leq c R+\frac{c}{R^{2}} \operatorname{tr}\left(u^{i j}\right) \quad \text { in } \Omega_{\delta},
\end{gathered}
$$

where $\operatorname{tr}\left(u^{i j}\right) \equiv \sum_{i=1}^{n} u^{i i}$.

Proof. For (33), by the assumption (8) and $C^{1}$ estimates of Lemma 5, we get

$$
\begin{aligned}
|T(u-\underline{u})| & \leq\left|(u-\underline{u})_{t}\right|+\left|(u-\underline{u})_{n}\right| \\
& \leq 2|D(u-\underline{u})| \\
& \leq 2\left|(u-\underline{u})_{\nu}\right|+2\left|(u-\underline{u})_{\tau}\right| \\
& \leq 2\left|(u-|x|)_{\tau}\right|+2\left|(\underline{u}-|x|)_{\tau}\right|+\frac{c}{R} \\
& \leq 2\left|u_{\tau}\right|+\frac{c}{R} \leq \frac{c}{\sqrt{R}},
\end{aligned}
$$

where $v=x /|x|$ and $\tau$ is unit vector orthogonal to $x$.

For the second inequality (34) we use that $(u-\underline{u})_{t}+(u-$ $\underline{u})_{n} \omega_{t}=0$ and note that $\omega_{t}(0)=0,\left|\omega_{i}\right| \leq c,\left|\omega_{i j}\right| \leq c / R$, $\left|\omega_{i j k}\right| \leq c / R^{2}$. Then for $x \in \partial B_{R}$,

$$
\begin{aligned}
T(u-\underline{u}) & =-\left(\omega_{t}-\sum_{\gamma=1}^{n-1} \omega_{t \gamma}(0) x_{\gamma}\right)(u-\underline{u})_{n} \\
& =-\sum_{\gamma, s=1}^{n-1} x_{\gamma} \omega_{t \gamma s}(\theta \widehat{x}) x_{s}(u-\underline{u})_{n}
\end{aligned}
$$

with $0<\theta<1$, which implies (34).
To prove (35), by Lemma 5 , we may take $1 /\left(1-|D u|^{2}\right)=$ $O(R)$. In view of (8), (30), and $u^{i j} u_{j k}=\delta_{i k}$, we obtain

$$
\begin{aligned}
\mid L T & (u-\underline{u}) \mid \\
= & \left|L\left((u-\underline{u})_{t}-\frac{x_{t}}{R}(u-\underline{u})_{n}\right)\right| \\
= & \mid u^{i j}\left[(u-\underline{u})_{t i j}-\left(\frac{x_{t}}{R}(u-\underline{u})_{n}\right)_{i j}\right] \\
& +\frac{n+2-\beta}{1-|D u|^{2}} u_{i}\left[(u-\underline{u})_{t i}-\left(\frac{x_{t}}{R}(u-\underline{u})_{n}\right)_{i}\right] \mid \\
\leq & \left|u^{i j} u_{t i j}+\frac{n+2-\beta}{1-|D u|^{2}} u_{i} u_{t i}\right| \\
& +\left|\frac{x_{t}}{R}\left(u^{i j} u_{n i j}+\frac{n+2-\beta}{1-|D u|^{2}} u_{i} u_{n i}\right)\right| \\
& +\left|u^{i j}\left(\underline{u}_{t i j}-\frac{x_{t}}{R} \underline{u}_{n i j}\right)\right|+\frac{n+2-\beta}{1-|D u|^{2}}\left|u_{i}\left(\underline{u}_{t i}+\frac{x^{t}}{R} \underline{u}_{n i}\right)\right| \\
& +\left|u^{i j}\left(\frac{\delta_{i t}}{R} u_{n j}+\frac{\delta_{j t}}{R} u_{n i}\right)\right|+\left|u^{i j}\left(\frac{\delta_{i t}}{R} \underline{u}_{n j}+\frac{\delta_{j t}}{R} \underline{u}_{n i}\right)\right| \\
& +\frac{n+2-\beta}{1-|D u|^{2}}\left|u_{i} \cdot \frac{\delta_{i t}}{R}(u-\underline{u})_{n}\right| \\
\leq & \cdot \operatorname{tr}\left(u^{i j}\right) \cdot\left(\left|D^{3} \underline{u}\right|+\frac{1}{R}\left|D^{2} \underline{u}\right|\right) \\
& +c \cdot \frac{n+2-\beta}{1-\left|D^{2} \underline{u}\right|^{2}}\left[\left|D^{2} \underline{u}\right|+\frac{1}{R^{2}}\right]+c \leq \frac{c}{R^{2}} \operatorname{tr}\left(\underline{u}^{i j}\right)+c .
\end{aligned}
$$

In the next lemma, we introduce a function $\mathscr{V}$, which will be the main part of a barrier function to prove Theorem 7 .

Lemma 9. There exists a positive constant $\varepsilon$ independent of $R$ such that

$$
\mathscr{V}:=(u-\underline{u})+\frac{1}{\sqrt{R}} d-\frac{1}{2 R^{5 / 4}} d^{2}
$$

fulfills the estimates

$$
\begin{gathered}
L \mathscr{V} \leq-\varepsilon R^{-(1 / n)(\beta-(7 / 4))}-\varepsilon R^{-(5 / 4)} \operatorname{tr}\left(u^{i j}\right), \quad \text { in } \Omega_{\delta}, \\
\mathscr{V} \geq 0, \quad \text { on } \partial \Omega_{\delta}
\end{gathered}
$$

provided that $\delta=R^{3 / 4}$ and $R$ is sufficiently large. Here $d=$ $R-|x|$ is the distance from $\partial B_{R}$.

Proof. In view of $\delta=R^{3 / 4}$, for $x \in \partial \Omega_{\delta}, d=R-|x| \leq \delta=R^{3 / 4}$, and $u \geq \underline{u}$, we have

$$
\mathscr{V}=(u-\underline{u})+\frac{1}{\sqrt{R}} d-\frac{1}{2 R^{5 / 4}} d^{2} \geq \frac{1}{\sqrt{R}} d-\frac{1}{2 R^{5 / 4}} d^{2} \geq 0
$$

on $\partial \Omega_{\delta}$. 
We fix $x \in \Omega_{\delta}$ and set $\nu=x /|x|$. Let $\tau, \tau^{\prime}$ belong to an orthonormal basis for the orthogonal complement of $\nu$ which we choose such that the submatrix $\left(\mathcal{u}^{\tau \tau^{\prime}}\right)$ is diagonal. Assume that $\nu$ and $\tau, \tau^{\prime}$ correspond to the indices $n$ and $1, \ldots, n-1$, respectively. We use the Einstein summation convention for $\tau, \tau^{\prime}$. The matrix $u^{i j}$ is positive, and thus testing with the vectors $\nu \pm \tau$ gives

$$
\left|u^{\nu \tau}\right| \leq \frac{1}{2}\left(u^{\nu v}+u^{\tau \tau}\right) .
$$

In view of

$$
\begin{gathered}
u^{\nu \nu}=u^{i j} \frac{x_{i}}{|x|} \frac{x_{j}}{|x|}, \\
\operatorname{tr}\left(u^{\tau \tau^{\prime}}\right)=\operatorname{tr}\left(u^{i j}\right)-u^{\nu v}=u^{i j}\left(\delta_{i j}-\frac{x_{i}}{|x|} \frac{x_{j}}{|x|}\right) .
\end{gathered}
$$

Direct computations using (17) give

$$
\begin{aligned}
L u & =u^{i j} u_{i j}+(n+2-\beta) \frac{|D u|^{2}}{1-|D u|^{2}} \\
& \leq(n+2-\beta) \frac{|D u|^{2}}{1-|D u|^{2}}+c .
\end{aligned}
$$

By (8), (16), and (42) we have

$$
\begin{aligned}
L \underline{u}= & u^{i j} \underline{u}_{i j}+\frac{n+2-\beta}{1-|D u|^{2}} u_{i} \underline{u}_{i} \\
= & \frac{n+2-\beta}{1-|D u|^{2}} u_{i} \underline{u}_{i}+u^{i j}\left[|x|_{i j}+(\underline{u}-|x|)_{i j}\right] \\
= & \frac{n+2-\beta}{1-|D u|^{2}} u_{i} \underline{u}_{i}+\frac{1}{|x|} u^{i j}\left(\delta_{i j}-\frac{x_{i}}{|x|} \frac{x_{j}}{|x|}\right)+u^{i j}(\underline{u}-|x|)_{i j} \\
= & \frac{n+2-\beta}{1-|D u|^{2}} u_{i} \underline{u}_{i}+\frac{1}{|x|} \operatorname{tr}\left(u^{\tau \tau^{\prime}}\right)+u^{\tau \tau^{\prime}}(\underline{u}-|x|)_{\tau \tau^{\prime}} \\
& +2 u^{\tau \nu}(\underline{u}-|x|)_{\tau \nu}+u^{\nu v}(\underline{u}-|x|)_{\nu v} \\
\geq & \frac{n+2-\beta}{1-|D u|^{2}} u_{i} \underline{u}_{i}+\left(\frac{1}{|x|}-\frac{c}{|x|^{2}}\right) \operatorname{tr}\left(u^{\tau \tau^{\prime}}\right)-\frac{c}{|x|^{2}} u^{\nu v}, \\
L d= & u^{i j}(R-|x|)_{i j}+\frac{n+2-\beta}{1-|D u|^{2}} u_{i}(R-|x|)_{i} \\
= & -\frac{1}{|x|} \operatorname{tr}\left(u^{\tau \tau^{\prime}}\right)-\frac{n+2-\beta}{1-|D u|^{2}} \frac{u_{i} x_{i}}{|x|}, \\
L d^{2}= & u^{i j}\left((R-|x|)^{2}\right)_{i j}+\frac{n+2-\beta}{1-|D u|^{2}} u_{i}\left((R-|x|)^{2}\right)_{i} \\
\geq & -\frac{2 R}{|x|} \operatorname{tr}\left(u^{\tau \tau^{\prime}}\right)+2 \operatorname{tr}\left(u^{i j}\right) \\
& -\frac{n+2-\beta}{1-|D u|^{2}} u_{i}\left(\frac{2 R x_{i}}{|x|}-2 x_{i}\right) \\
= & -2 \frac{d}{|x|} \operatorname{tr}\left(u^{\tau \tau^{\prime}}\right)+2 u^{\nu v}-\frac{n+2-\beta}{1-|D u|^{2}} u_{i}\left(\frac{2 R x_{i}}{|x|}-2 x_{i}\right) .
\end{aligned}
$$

Then,

$$
\begin{aligned}
L \mathscr{V}= & L(u-\underline{u})+\frac{1}{\sqrt{R}} L d-\frac{1}{2 R^{5 / 4}} L d^{2} \\
\leq & c+\frac{n+2-\beta}{1-|D u|^{2}} u_{i}\left(u_{i}-\underline{u}_{i}\right) \\
& +\frac{n+2-\beta}{1-|D u|^{2}} \frac{u_{i} x_{i}}{R^{5 / 4}|x|}\left(d-R^{3 / 4}\right) \\
& -\left[\frac{1}{|x|}-\frac{c}{|x|^{2}}+\frac{1}{|x|}\left(\frac{1}{\sqrt{R}}-\frac{d}{R^{5 / 4}}\right)\right] \operatorname{tr}\left(u^{\tau \tau^{\prime}}\right) \\
& -\left(\frac{1}{R^{5 / 4}}-\frac{c}{|x|^{2}}\right) u^{\nu v} .
\end{aligned}
$$

Thus, for $R$ large enough, we have

$$
L \mathscr{V} \leq c R^{1 / 2}-\frac{1}{2 R} \operatorname{tr}\left(u^{\tau \tau^{\prime}}\right)-\frac{1}{2 R^{5 / 4}} u^{\nu \nu} .
$$

Expanding the determinant and using that $\left(u^{\tau \tau^{\prime}}\right)$ is a diagonal matrix give

$$
\begin{aligned}
\operatorname{det}\left(u^{i j}\right) & =\operatorname{det}\left(\begin{array}{ccccc}
u^{11} & 0 & \cdots & 0 & u^{1 n} \\
0 & \ddots & \ddots & \vdots & \vdots \\
\vdots & \ddots & \ddots & 0 & \vdots \\
0 & \cdots & 0 & u^{n-1 n-1} & u^{n-1 n} \\
u^{1 n} & \cdots & \cdots & u^{n-1 n} & u^{n n}
\end{array}\right) \\
& =\prod_{i} u^{i i}-\sum_{\tau}\left|u^{n \tau}\right|^{2} \prod_{\tau^{\prime} \neq \tau} u^{\tau^{\prime} \tau^{\prime}} \leq \prod_{i} u^{i i} .
\end{aligned}
$$

By the inequality for arithmetic and geometric means,

$$
\begin{aligned}
& \frac{1}{R} \operatorname{tr}\left(u^{\tau \tau^{\prime}}\right)+\frac{1}{R^{5 / 4}} u^{\nu \nu} \\
& \quad \geq n\left[\left(\frac{1}{R}\right)^{n-1} \cdot \frac{1}{R^{5 / 4}} \prod_{i} u^{i i}\right]^{1 / n} \\
& \quad \geq n\left[\left(\operatorname{det}\left(u_{i j}\right)\right)\right]^{-(1 / n)} R^{-(1 / n)(n+(1 / 4))} .
\end{aligned}
$$

Hence for large $R$,

$$
\begin{aligned}
L \mathscr{V} \leq & c R^{1 / 2}-\frac{1}{4 R} \operatorname{tr}\left(u^{\tau \tau^{\prime}}\right)-\frac{1}{4 R^{5 / 4}} u^{\nu \nu}-\frac{1}{4 R} \operatorname{tr}\left(u^{\tau \tau^{\prime}}\right) \\
& -\frac{1}{4 R^{5 / 4}} u^{\nu \nu} \\
\leq & c R^{1 / 2}-c\left[\operatorname{det}\left(u_{i j}\right)\right]^{-(1 / n)} R^{-(1 / n)(n+(1 / 4))}-\frac{1}{4 R^{5 / 4}} \operatorname{tr}\left(u^{i j}\right) \\
= & c R^{1 / 2}-c\left[\left(1-|D u|^{2}\right)^{(n+2-\beta) / 2}\right]^{-(1 / n)} R^{-(1 / n)(n+(1 / 4))} \\
& -\frac{1}{4 R^{5 / 4}} \operatorname{tr}\left(u^{i j}\right)
\end{aligned}
$$




$$
\begin{aligned}
& \leq c R^{1 / 2}-c R^{(3-2 \beta-2 n) / 4 n}-\frac{1}{4 R^{5 / 4}} \operatorname{tr}\left(u^{i j}\right) \\
& \leq-c R^{(3-2 \beta-2 n) / 4 n}-\frac{1}{4 R^{5 / 4}} \operatorname{tr}\left(u^{i j}\right) .
\end{aligned}
$$

Note that we have used the fact $(3-2 \beta-2 n) / 4 n>1 / 2$ in the last inequality, which is from the assumption $\beta<(3 / 2)-$ $2 n$.

Lemma 10. There exists a positive constant A independent of $R$ such that

$$
\Theta:=\mathscr{V}+A \cdot \frac{1}{R^{2}} \cdot\left|x-x_{0}\right|^{2} \pm T(u-\underline{u})
$$

satisfies

$$
\begin{aligned}
& L \Theta \leq 0, \quad \text { in } \Omega_{\delta}, \\
& \Theta \geq 0, \quad \text { on } \partial \Omega_{\delta},
\end{aligned}
$$

where $\delta=R^{3 / 4}$ and $\mathscr{V}$ is as in Lemma 9 .

Proof. According to Lemma 9, the fact $\Theta \geq 0$ on $\partial \Omega_{\delta}$ follows from

$$
A \cdot \frac{1}{R^{2}} \cdot\left|x-x_{0}\right|^{2} \pm T(u-\underline{u}) \geq 0 \quad \text { on } \partial \Omega_{\delta},
$$

which can be attained by choosing $A$ sufficiently large. The property $L \Theta \leq 0$ now follows from the inequality

$$
\begin{aligned}
& -\varepsilon R^{(3-2 \beta-2 n) / 4 n}-\varepsilon R^{-5 / 4} \operatorname{tr}\left(u^{i j}\right)+c A R^{-(1 / 4)}+c \\
& +c \cdot \frac{1+A}{R^{2}} \operatorname{tr}\left(u^{i j}\right) \leq 0,
\end{aligned}
$$

which holds for $R$ large enough.

Proof of Theorem 7. The maximum principle applied to (52) yields that $\Theta \geq 0$ in $\Omega_{\delta}$. Since $\Theta\left(x_{0}\right)=0$, it follows that

$$
\Theta_{\nu}\left(x_{0}\right) \geq 0
$$

with $v=-x_{0} /\left|x_{0}\right|$. Thus we get

$$
\mathscr{V}_{\nu}\left(x_{0}\right) \geq\left|(T(u-\underline{u}))_{\nu}\right|\left(x_{0}\right) .
$$

That is,

$$
\begin{gathered}
{\left[-(u-\underline{u})_{n}-\frac{1}{\sqrt{R}}(R-|x|)_{n}+\frac{1}{R^{5 / 4}}(R-|x|)_{n}^{2}\right]\left(x_{0}\right)} \\
\geq\left|(u-\underline{u})_{t n}+\frac{x_{t}}{R}(u-\underline{u})_{n n}\right|\left(x_{0}\right)=\left|(u-\underline{u})_{t n}\right|\left(x_{0}\right),
\end{gathered}
$$

which, together with (8) and (14), implies

$$
\left|u_{t n}\left(x_{0}\right)\right| \leq\left|\underline{u}_{t n}\left(x_{0}\right)\right|+\left|(u-\underline{u})_{n}\right|+\frac{1}{\sqrt{R}} \leq \frac{c}{\sqrt{R}} \text {. }
$$

That is, (28) holds.
Theorem 11 (double normal $C^{2}$-estimates at the outer boundary). Under the assumption of Theorem 2 and the notation of Theorem 7, we have

$$
\left|u_{v v}\left(x_{0}\right)\right| \leq c .
$$

Proof. As the proof of Lemma 9, we fix $x_{0} \in \partial B_{R}$ and set $\nu=x_{0} /\left|x_{0}\right|$. Let $\tau, \tau^{\prime}$ belong to an orthonormal basis for the orthogonal complement of $v$ which we choose such that the submatrix $\left(u^{\tau \tau^{\prime}}\right)$ is diagonal. Assume that $\nu$ and $\tau, \tau^{\prime}$ correspond to the indices $n$ and $1, \ldots, n-1$, respectively. We expand the determinant,

$$
\begin{aligned}
(1 & \left.-|D u|^{2}\right)^{(n+2-\beta) / 2} \\
& =\operatorname{det}\left(u_{i j}\right)=u_{n n} \cdot \prod_{i<n} u_{i i}-\sum_{k<n} u_{k n}^{2} \cdot \prod_{k \neq i<n} u_{i i} \\
& =u_{n n} \cdot \prod_{i<n} u_{i i}-\prod_{i<n} u_{i i} \sum_{k<n} u_{k n}^{2} \frac{1}{u_{k k}} .
\end{aligned}
$$

Then, for $\beta<(3 / 2)-2 n$, we have

$$
\begin{aligned}
u_{n n} & =\frac{\left(1-|D u|^{2}\right)^{(n+2-\beta) / 2}}{\prod_{i<n} u_{i i}}+\sum_{k<n} \frac{u_{k n}^{2}}{u_{k k}} \\
& \leq \frac{c R^{-((n+2-\beta) / 2)}}{(c / R)^{n-1}}+\sum_{k<n} \frac{(c / \sqrt{R})^{2}}{c / R} \leq c .
\end{aligned}
$$

Proof of Theorem 2. It follows from Theorems 6, 7, and 11 that $\left\|u^{R}\right\|_{C^{2}}$ are uniformly bounded in $R$. By the standard Krylov/Shafanov theory, Schauder regularity theory, and a diagonal sequence argument, we obtain a smooth locally strictly convex solution $u$ to (3)-(5) on exterior domain $\mathbb{R}^{n} \backslash$ D.

\section{Proof of Theorem 3}

In this section, we prove Theorem 3, which gives a simple example of a barrier construction.

Proof of Theorem 3. We introduce functions

$$
\begin{gathered}
\varphi(\tau)=a \rho_{0}^{2} \tau^{-3} \\
\eta(r)=-\int_{\rho_{0}}^{r}\left(\int_{\rho}^{\infty} \varphi(\tau) d \tau\right) d \rho
\end{gathered}
$$

where $0<a<1$ will be determined. We define $\underline{u}$ by

$$
\begin{gathered}
\underline{u}: \mathbb{R}^{n} \backslash B_{\rho_{0}} \longrightarrow \mathbb{R} \\
x \longmapsto|x|-\rho_{0}+\eta(|x|) .
\end{gathered}
$$

Then, for $r \geq \rho_{0}$,

$$
0<-\eta^{\prime}(r)=\int_{r}^{\infty} \varphi(\tau) d \tau=\frac{a}{2} \rho_{0}^{2} r^{-2} \leq \frac{a}{2}<1 .
$$


Obviously, $\underline{u}=0$ on $\partial B_{\rho_{0}}$. Moreover,

$$
\begin{gathered}
\sup |\underline{u}-r| \leq \rho_{0}+\int_{\rho_{0}}^{\infty}\left(\int_{\rho}^{\infty} \varphi(\tau) d \tau\right) d \rho, \\
|D(\underline{u}-r)|=\left|\eta^{\prime}(r)\right|=O\left(\frac{1}{r}\right), \\
\left|D^{2}(\underline{u}-r)\right|+\left|D^{3} \underline{u}\right|=O\left(\frac{1}{r^{2}}\right),
\end{gathered}
$$

where $r=|x|$. Therefore, $u$ is close to a cone in the sense of (7) and satisfies the regularity conditions (8) and (17).

We compute the Gauss curvature of graph $\underline{u}$ as follows:

$$
\begin{gathered}
\underline{u}_{i}=\left(1+\eta^{\prime}\right) \frac{x_{i}}{r}, \quad|D \underline{u}|=1+\eta^{\prime}, \\
\underline{u}_{i j}=\left(1+\eta^{\prime}\right) \frac{1}{r}\left(\delta_{i j}-\frac{x_{i} x_{j}}{r^{2}}\right)+\eta^{\prime \prime}(r) \frac{x_{i} x_{j}}{r^{2}} \\
\operatorname{det} D^{2} \underline{u}=\frac{\eta^{\prime \prime}(r)}{r^{n-1}}\left(1+\eta^{\prime}\right)^{n-1}=\frac{\varphi}{r^{n-1}}\left(1+\eta^{\prime}\right)^{n-1} .
\end{gathered}
$$

Take $0<a<\min \left\{1,1 /\left(2 \rho_{0}\right)^{2}\right\}$. Using the assumption $\beta<0$ and the fact that

$$
|D \underline{u}|^{2}=\left(1+\eta^{\prime}\right)^{2}=1-a \rho_{0}^{2} r^{-2}+\frac{a^{2}}{4} \rho_{0}^{4} r^{-4} \geq 1-a \rho_{0}^{2} r^{-2},
$$

we conclude that

$$
\begin{aligned}
& \frac{\operatorname{det} D^{2} \underline{u}}{\left(1-|D \underline{u}|^{2}\right)^{(n+2-\beta) / 2}} \\
& =\varphi(r)\left(1+\eta^{\prime}\right)^{n-1} \cdot \frac{1}{\left(1-\left(1+\eta^{\prime}\right)^{2}\right)^{(n+2-\beta) / 2}} \cdot \frac{1}{r^{n-1}} \\
& \geq a \rho_{0}^{2} r^{-3} 2^{1-n}\left(a \rho_{0}^{2} r^{-2}\right)^{-(n+2-\beta) / 2} \cdot r^{1-n} \\
& =2^{1-n} a^{-(n+\beta) / 2} \rho_{0}^{\beta-n} r^{-\beta} \geq\left(2 \sqrt{a} \rho_{0}\right)^{-n} \geq 1 .
\end{aligned}
$$

Thus,

$$
\begin{gathered}
\operatorname{det} D^{2} \underline{u} \geq\left(1-|D \underline{u}|^{2}\right)^{(n+2-\beta) / 2}, \quad|x|>\rho_{0}, \\
\underline{u}=0, \quad|x|=\rho_{0} .
\end{gathered}
$$

The theorem is completed.

\section{Conflict of Interests}

The author declares that there is no conflict of interests regarding the publication of this paper.

\section{Acknowledgments}

This work is supported by the National Natural Science Foundation of China (11301034, 11201011, and 11391240188) and the Fundamental Research Funds for the Central Universities (2013RC0901).

\section{References}

[1] P. Bayard and O. C. Schnürer, "Entire spacelike hypersurfaces of constant Gauß curvature in Minkowski space," Journal für die reine und angewandte Mathematik, vol. 627, pp. 1-29, 2009.

[2] B. Guan, H. Jian, and R. M. Schoen, "Entire spacelike hypersurfaces of prescribed Gauss curvature in Minkowski space," Journal für die Reine und Angewandte Mathematik, vol. 595, pp. 167-188, 2006.

[3] M. A. S. Aarons, "Mean curvature flow with a forcing term in Minkowski space," Calculus of Variations and Partial Differential Equations, vol. 25, no. 2, pp. 205-246, 2006.

[4] K. Ecker, "Interior estimates and longtime solutions for mean curvature flow of noncompact spacelike hypersurfaces in Minkowski space," Journal of Differential Geometry, vol. 46, no. 3, pp. 481-498, 1997.

[5] K. Ecker and G. Huisken, "Parabolic methods for the construction of spacelike slices of prescribed mean curvature in cosmological spacetimes," Communications in Mathematical Physics, vol. 135, no. 3, pp. 595-613, 1991.

[6] Y. Liu and H. Jian, "Evolution of spacelike hypersurfaces by mean curvature minus external force field in Minkowski space," Advanced Nonlinear Studies, vol. 9, no. 3, pp. 513-522, 2009.

[7] H. Jian, "Translating solitons of mean curvature flow of noncompact spacelike hypersurfaces in Minkowski space," Journal of Differential Equations, vol. 220, no. 1, pp. 147-162, 2006.

[8] H. Ju, J. Lu, and H. Jian, "Translating solutions to mean curvature flow with a forcing term in Minkowski space," Communications on Pure and Applied Analysis, vol. 9, no. 4, pp. 963-973, 2010.

[9] H. Ju, J. Bao, and H. Jian, "Existence for translating solutions of Gauss curvature flow on exterior domains," Nonlinear Analysis: Theory, Methods \& Applications, vol. 75, no. 8, pp. 3629-3640, 2012.

[10] F. Finster and O. C. Schnürer, "Hypersurfaces of prescribed Gauss curvature in exterior domains," Calculus of Variations and Partial Differential Equations, vol. 15, no. 1, pp. 67-80, 2002.

[11] Y. Huang, H. Jian, and N. Su, "Spacelike hypersurfaces of prescribed Gauss-Kronecker curvature in exterior domains," Acta Mathematica Sinica, vol. 25, no. 3, pp. 491-502, 2009.

[12] B. Guan, “The Dirichlet problem for Monge-Ampère equations in non-convex domains and spacelike hypersurfaces of constant Gauss curvature," Transactions of the American Mathematical Society, vol. 350, no. 12, pp. 4955-4971, 1998.

[13] D. Gilbarg and N. S. Trudinger, Second Order Elliptic Partial Differential Equations, Springer, Berlin, Germany, 2nd edition, 1983. 


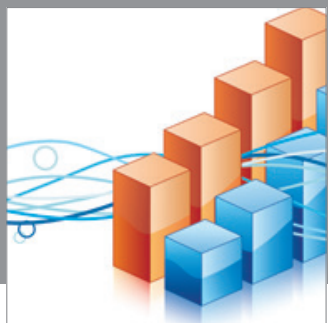

Advances in

Operations Research

mansans

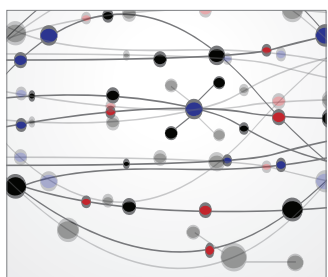

The Scientific World Journal
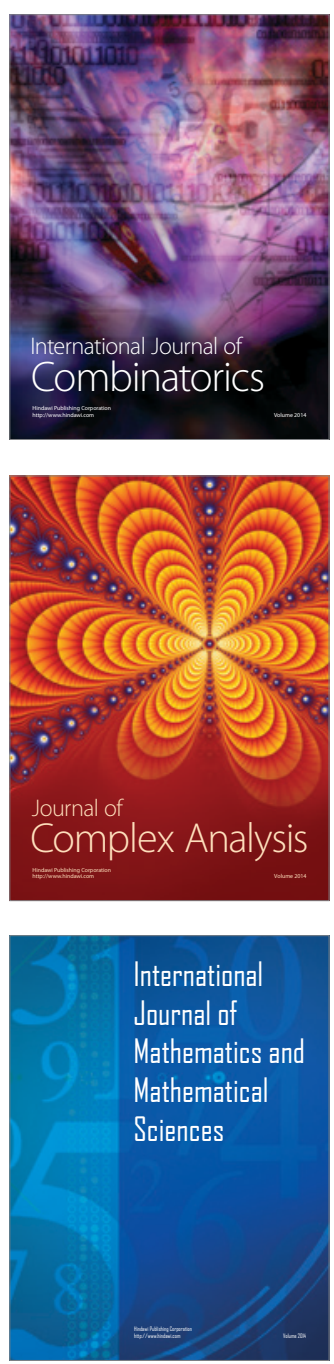
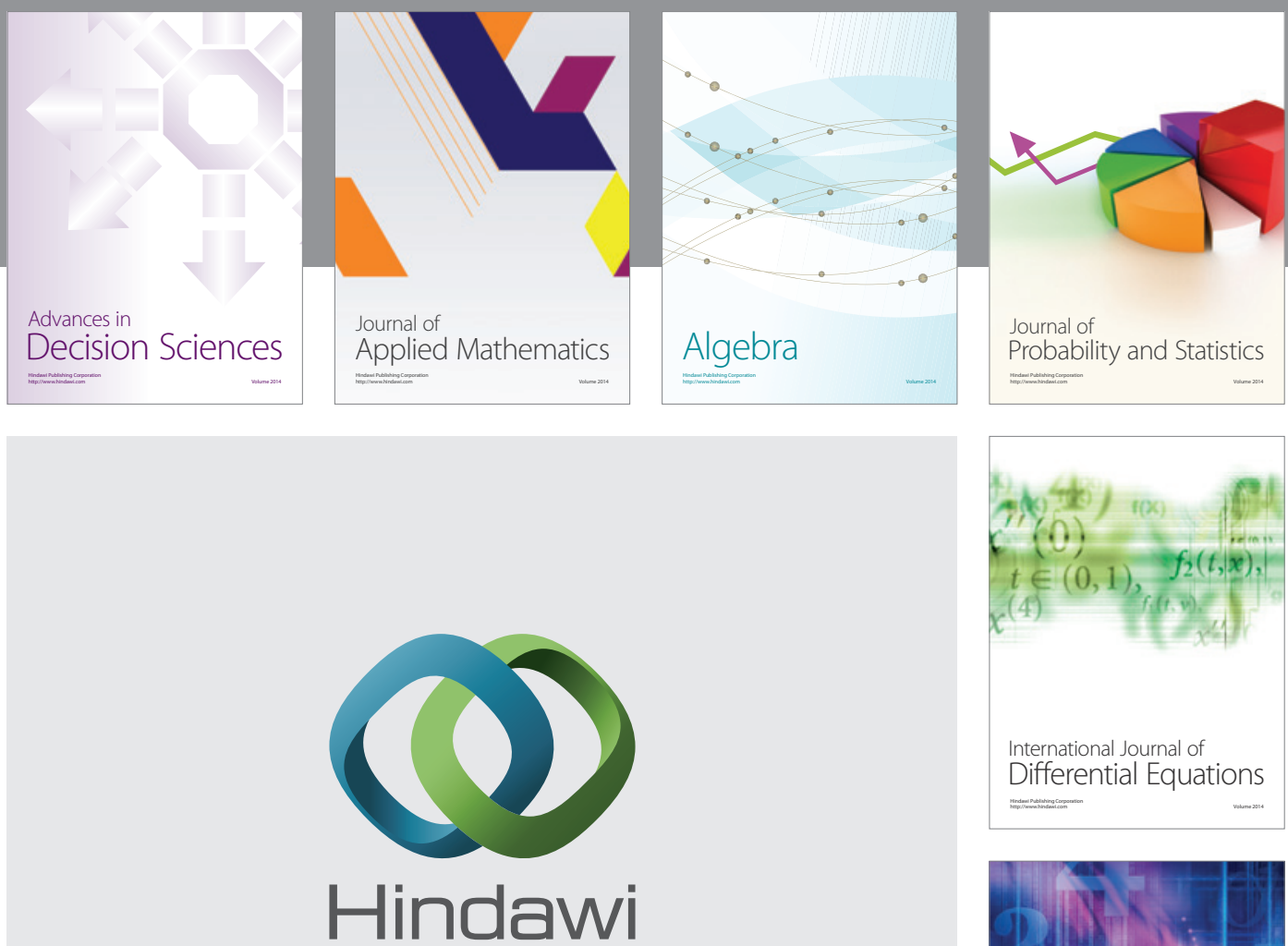

Submit your manuscripts at http://www.hindawi.com
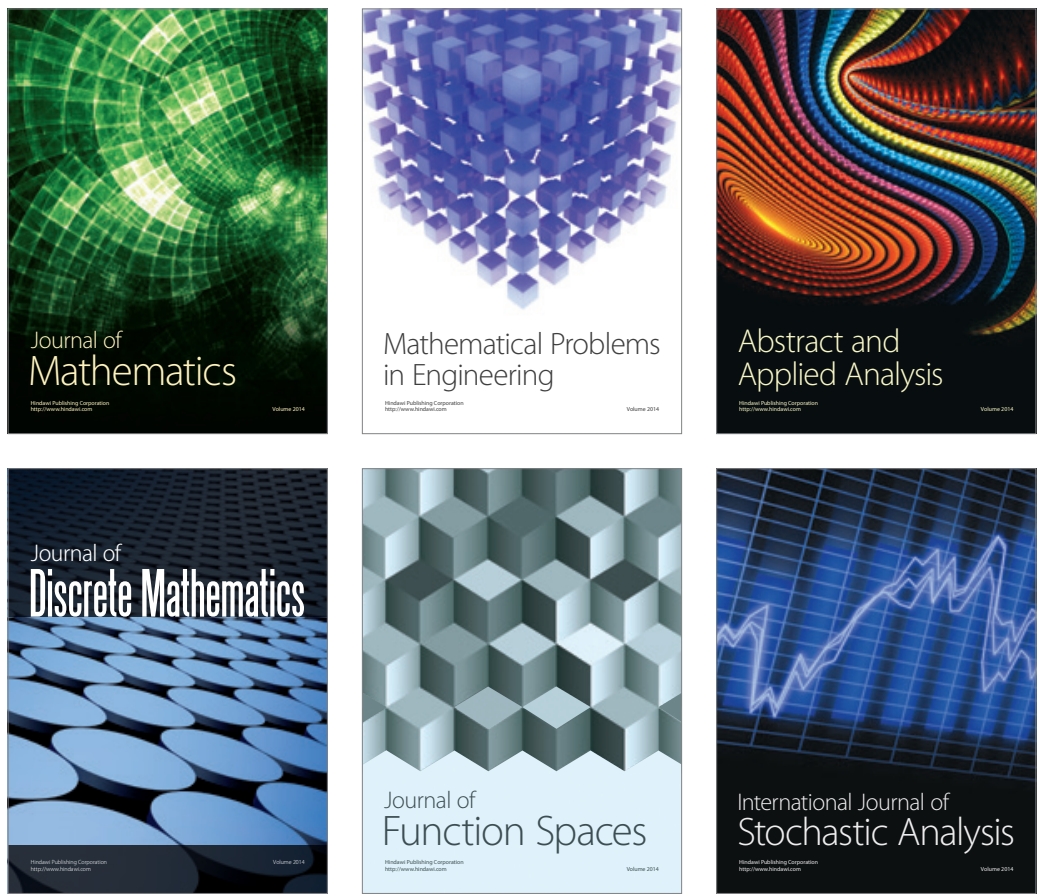

Journal of

Function Spaces

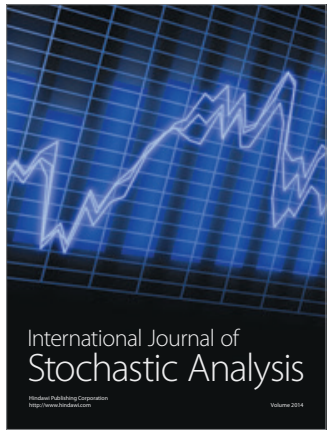

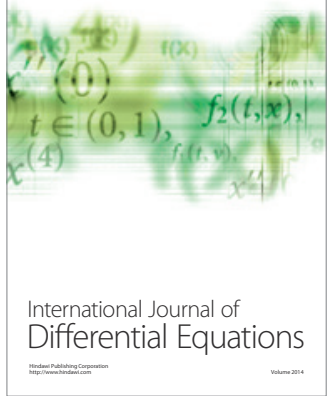
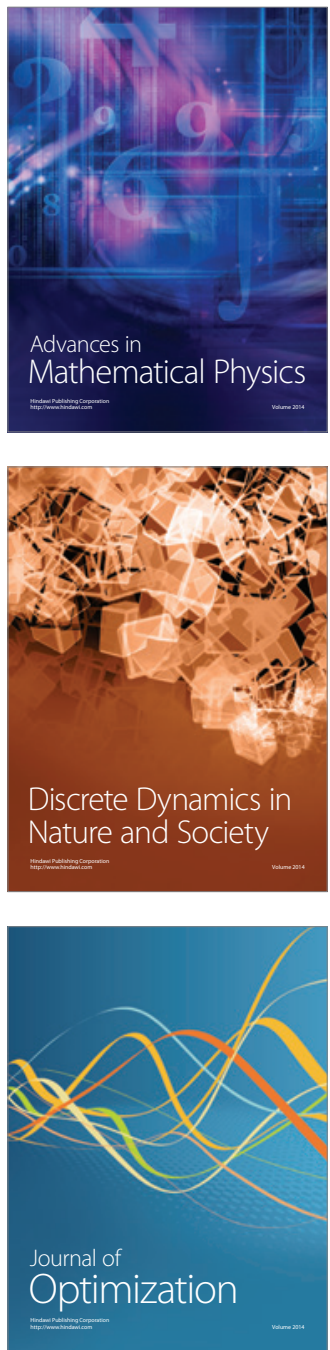